\title{
High-Concentrate Diets and Polyunsaturated Oils Alter Trans and Conjugated Isomers in Bovine Rumen, Blood, and Milk
}

\author{
J. J. Loor, ${ }^{\star}$ A. Ferlay, A. Ollier, K. Ueda, $†$ M. Doreau, and Y. Chilliard \\ Herbivore Research Unit INRA-Theix, 63122 St-Genès Champanelle, France
}

\section{ABSTRACT}

Three Holstein cows were fed a high-concentrate diet (65:35 concentrate to forage) supplemented with either $5 \%$ sunflower oil (SO), $5 \%$ linseed oil (LO), or $2.5 \%$ fish oil (FO) to examine effects on biohydrogenation and fatty acid profiles in rumen, blood plasma, and milk. Diets were fed in a $3 \times 3$ Latin square with 4 -wk periods with grass hay as the forage. Milk yield, dry matter intake, and percentages of milk fat (2.64) and protein (3.22) did not differ. All diets resulted in incomplete hydrogenation of unsaturated fatty acids as indicated by the profiles of 18:1 isomers, conjugated 18:2 isomers, nonconjugated 18:2 isomers, and 18:0 in ruminal fluid. Percentages of 8:0-14:0 and 16:0 in milk fat were greater with FO. Percentage and yield of trans10,cis1218:2 were small and greater in cows fed SO $(0.14 \%$, $0.57 \mathrm{~g} / \mathrm{d})$ than FO $(0.03 \%, 0.15 \mathrm{~g} / \mathrm{d})$ or $\mathrm{LO}(0.04 \%, 0.12$ $\mathrm{g} / \mathrm{d})$. Percentage and yield of trans 10-18:1, however, increased with FO $(6.16 \%)$ and SO $(6.47 \%)$ compared with LO (1.65\%). Dietary FO doubled percentage of cis11-18:1 in rumen, plasma, and milk fat. Despite a lack of difference in ruminal percentage of trans 11-18:1 (10.5\%), cows fed FO had greater plasma trans11-18:1 (116 vs. $61.5 \mu \mathrm{g} / \mathrm{mL}$ ) but this response did not result in greater trans11-18:1 percentage in milk fat, which averaged $5.41 \%$ across diets. Percentage $(2.2 \%)$ and yield $(14.3 \mathrm{~g} / \mathrm{d})$ of cis 9 ,trans 11-18:2 in milk fat did not differ due to oils. Unique responses to feeding LO included greater than 2 -fold increases in percentages of trans $13+14-18: 1$, trans $15-18: 1$, trans $16-18: 1$, cis 15 18:1, cis 9 ,trans 12-18:2 and trans 11, cis 15 -18:2 in umen, plasma, and milk, and cis9,trans13-18:2 in plasma and milk. Ruminal 18:0 percentage had the highest positive correlation with milk fat content $(\mathrm{r}=0.82)$ across all diets. When compared with previous data with cows

Received May 4, 2005.

Accepted July 14, 2005.

Corresponding author: Yves Chilliard; e-mail: yves.chilliard@ clermont.inra.fr.

*Current address: Department of Animal Sciences, University of Illinois, 206 ERML, Urbana, IL, 61801 (E-mail: jloor@uiuc.edu).

$\dagger$ Current address: Graduate School of Agriculture, Hokkaido University, Sapporo, 060-8589, Japan (E-mail: ueko@anim.agr.hokudai.ac.jp). fed high-concentrate diets without oil supplementation, results suggest that greater production of trans 10-18:1, cis 11-18:1, and trans 11,cis15-18:2 coupled with low production of 18:0 in the rumen may be associated with low milk fat content when feeding high-concentrate diets and fish oil. In contrast, SO or LO could lead to low milk fat content by increasing ruminal trans 10 18:1 (SO) or trans 11,cis 15-18:2 and trans9,trans 12-18:2 (LO) along with a reduction in mammary synthesis of 8:0-16:0. Simultaneous increases in ruminal trans1118:1 with fish oil, at a fraction of sunflower oil supplementation, may represent an effective strategy to maintain cis 9 ,trans 11-18:2 synthesis in mammary while reducing milk fat output and sparing energy.

(Key words: high-concentrate diet, polyunsaturated oil, trans fatty acid, milk conjugated linoleic acid)

Abbreviation key: CLA = conjugated linoleic acids, DHA = docosahexaenoic acid, EPA = eicosapentaenoic acid, FO = high-concentrate diet plus $2.5 \%$ fish oil, $\mathbf{L O}=$ high-concentrate diet plus 5\% linseed oil, SO = highconcentrate diet plus 5\% sunflower oil.

\section{INTRODUCTION}

Extensive literature over the past few decades suggests that milk fat depression is a multifactorial phenomenon (see review by Bauman and Griinari, 2003). Acetate and butyrate production $(\mathrm{mol} / \mathrm{d})$ are decreased by high dietary concentrate (Sutton et al., 2003), partly accounting for lower jugular plasma concentration and jugular-mammary venous difference for acetate and BHBA (Loor et al., 2005a). These changes may contribute to the effect of dietary concentrate level on milk fat yield. Others, however, have recently shown that despite an estimated $34 \%$ more acetate and $35 \%$ more butyrate produced in the rumen of cows fed high-concentrate diets there was a significant depression in milk fat yield (Oba and Allen, 2003). Recent studies clearly suggest that unique fatty acid intermediates produced by altered ruminal biohydrogenation are causally related to diet-induced milk fat depression (Bauman and Griinari, 2003).

Some of the greatest reductions in milk fat yield have been observed with high-concentrate diets supple- 
mented with vegetable or marine oils (Griinari et al., 1998; Piperova et al., 2000; Peterson et al., 2003; Loor et al., 2005a,b). Complete ruminal biohydrogenation of polyunsaturated fatty acids is reduced under these circumstances resulting in higher intestinal flows of trans-18:1 isomers, conjugated linoleic acids (CLA), and other nonconjugated 18:2 isomers (Piperova et al., 2002; Shingfield et al., 2003; Loor et al., 2004b). Vegetable oils rich in linoleic acid fed with a high-concentrate diet resulted in higher percentages of trans 10-18:1 in milk fat (Griinari et al., 1998; Piperova et al., 2000; Peterson et al., 2003). Cis9,trans11-18:2, the primary CLA in milk fat, also increased along with small significant increases in trans7,cis9-18:2 (Piperova et al., 2000) and trans10,cis12-18:2 (Piperova et al., 2000; Peterson et al., 2003).

Cis9,trans11-CLA and vaccenic acid (its precursor in tissues) in meat and milk are examples of biohydrogenation intermediates that may have beneficial implications for human health (Corl et al., 2003). Alterations in biohydrogenation pathways due to diet are important because increased ruminal production of trans 10-18:1 and trans 10, cis 12-18:2 have been associated with milk fat depression (Bauman and Griinari, 2003), and because a reduction in trans 11-18:1 production in the rumen may decrease endogenous synthesis of cis9,trans11-18:2 in tissues. Furthermore, trans11-18:1 in milk fat was transiently increased for $1 \mathrm{wk}$ after addition of polyunsaturated oils to high-concentrate or corn silage-based diets, after which it decreased along with a simultaneous increase in trans 10-18:1 (Chilliard and Ferlay, 2004; Roy et al., 2005).

Previous studies compared effects of TMR containing mixtures of fish oil and different sources of unsaturated fatty acids (AbuGhazaleh et al., 2003), or concentrate:forage ratio and linseed oil (Loor et al., 2005a) fed to dairy cows on blood plasma metabolites, milk composition, and profiles of trans-18:1 and CLA isomers in blood plasma and milk fat. Furthermore, previous results suggest that the nature of supplemental polyunsaturated fatty acids added to high-concentrate diets likely alters the profile and amount of hydrogenation intermediates available for secretion in milk. Thus, the primary objective of this study was to evaluate the effectiveness of high-concentrate diets in combination with fish oil [ $20 \mathrm{~g} / 100 \mathrm{~g}$ of total fatty acids 20:5n-3 (eicosapentaenoic acid, EPA) and $7 \mathrm{~g} / 100 \mathrm{~g}$ of $22: 6 \mathrm{n}-3$ (docosahexaenoic acid, DHA)], linseed oil (51 g/100 g 18:3n-3), or sunflower oil (69 g/100 g 18:2n-6) in modifying ruminal, blood plasma, and milk fat cis and trans isomers of 18:1, nonconjugated 18:2, and conjugated 18:2 isomers. In addition, we studied the correlations between these isomers in ruminal fluid and milk fat content.

\section{MATERIALS AND METHODS}

\section{Animals and Diets}

Three lactating multiparous Holstein cows, $84 \pm 21$ DIM, and cannulated in the rumen and duodenum were used in a $3 \times 3$ Latin square design during three 4 -wk periods to evaluate responses to feeding diets with a high (65:35) concentrate to forage (grass hay) ratio supplemented (on a DM basis) with $2.5 \%$ fish oil (FO), $5.0 \%$ linseed oil (LO), or 5.0\% sunflower oil (SO) (Loor et al., 2005c). The level of fish oil was chosen for the following reasons: 1) to avoid possible detrimental effects on DMI, which are proportional to the amount fed (Doreau and Chilliard, 1997; Donovan et al., 2000); 2) to avoid excessive decreases in milk fat yield (Chilliard et al., 2001); and 3) to allow intake of EPA plus DHA at less than $25 \%$ the amount of linoleic plus linolenic acid in cows receiving $\mathrm{SO}$ or $\mathrm{LO}$, which in a previous in vitro study was as effective in elevating trans11-18:1 as linoleic acid (AbuGhazaleh and Jenkins, 2004). To allow cows to adapt to oil-supplemented diets, all cows were fed a diet supplemented with an oil mixture comprising sunflower oil, linseed oil, plus fish oil ( $40 \mathrm{~g} / \mathrm{d}+40 \mathrm{~g} / \mathrm{d}$ $+20 \mathrm{~g} / \mathrm{d}$, respectively) for $3 \mathrm{wk}$ before the first experimental period. Cows were housed in a tie-stall barn during the experiment. The concentrate mixture with oils (homogeneously mixed) was prepared daily and, along with the forage, was offered in equal amounts at 0900, 1330, and $1700 \mathrm{~h}$ for ad libitum consumption. Hay and concentrate were given separately. The desired forage:concentrate ratio was maintained by daily adjustment of forage and concentrate amounts offered, depending on the refusals of the previous day.

The concentrate mixture contained (DM basis) on average $28.2 \%$ beet pulp, $18.9 \%$ ground wheat, $18.9 \%$ barley, $14.2 \%$ rapeseed meal, $13.7 \%$ soybean meal, $2.0 \%$ wet beet molasses, $3.1 \%$ buffers, $0.5 \%$ binding agent, and $0.5 \%$ mineral-vitamin mix. Cows were milked at 0600 and $1700 \mathrm{~h}$.

\section{Sampling, Measurements, and Analyses}

Ruminal fluid ( $300 \mathrm{~mL}$ ) was collected during wk 4 by suction from the ventral sac via the ruminal cannula at $0900,1100,1300,1500,1700,2000$, and $0000 \mathrm{~h}$ (Loor et al., 2004b). Milk production and DMI were recorded daily throughout the experiment. Milk was sampled at each milking during the last $5 \mathrm{~d}$ of wk 4 . One $50-\mathrm{mL}$ aliquot from each of these milkings containing potassium bichromate (Merck, Fontenay-Sus-Bois, France) was stored at $4^{\circ} \mathrm{C}$ until analyzed for fat, protein, and lactose by infrared analysis with a 3-channel spectrophotometer (AOAC, 1997; CILAL, Theix, France). Additional 3-mL aliquots from 2 consecutive milkings 
on the last day of wk 4 were also collected and stored at $-20^{\circ} \mathrm{C}$ before isolation of milk fat and fatty acid analysis. These samples were composited based on a.m. and p.m. milk production. Data on milk production and DMI were averaged over the last $5 \mathrm{~d}$ of wk 4 before statistical analysis.

For plasma total fatty acid analysis, blood samples $(50 \mathrm{~mL})$ were collected in EDTA-containing Vacutainer tubes (CML, Nemours, France) from the jugular vein at $0830 \mathrm{~h}$ on the last day of wk 4 . For plasma metabolite analysis, an additional $10 \mathrm{~mL}$ of blood was collected from the jugular and abdominal mammary vein by venipuncture using EDTA-containing $(0.47 \mathrm{~mol} / \mathrm{L})$ Vacutainers. Blood was centrifuged at $3000 \times \mathrm{g}$ for $15 \mathrm{~min}$ for harvesting plasma. Plasma was stored at $-20^{\circ} \mathrm{C}$ until analyzed for fatty acids and metabolites. Plasma concentrations of metabolites were determined as described by Ferlay and Chilliard (1999) with an ELAN autoanalyzer using the same kits and procedures as in Loor et al. (2005a). Insulin and leptin were determined by radioimmunoassay as described in Delavaud et al. (2002).

Plasma total lipids were extracted with chloroform/ methanol (2:1, vol/vol; Loor et al., 2005a). Fatty acids in plasma lipids were methylated with $2 \mathrm{~mL}$ of $0.5 \mathrm{~N}$ $\mathrm{NaOCH}_{3}$ at $50^{\circ} \mathrm{C}$ for $30 \mathrm{~min}$, followed by $2 \mathrm{~mL}$ of $14 \%$ boron trifluoride in methanol at $50^{\circ} \mathrm{C}$ for $30 \mathrm{~min}$ (Loor et al., 2005a). Tricosanoate (Sigma, Saint-Quentin Fallavier, France) was used as the internal standard. Fatty acids in milk fat were directly methylated with $1 \mathrm{~mL}$ of $2 \mathrm{~N}$ methanolic $\mathrm{NaOCH} 3$ at room temperature for $20 \mathrm{~min}$, followed by $1 \mathrm{~mL}$ of $14 \%$ boron trifluoride in methanol at room temperature for $20 \mathrm{~min}$ (Loor et al., 2005a). Fatty acids in mixed ruminal fluid were methylated with the same protocol as for duodenal fatty acids (Loor et al., 2004b, 2005c). In all cases, fatty acid methyl esters were recovered in $1 \mathrm{~mL}$ of hexane. Samples were injected by autosampler into a Trace-GC 2000 Series gas chromatograph equipped with a flame-ionization detector (Thermo Finnigan, Les Ulis, France). Methyl esters from all samples were separated on a $100 \mathrm{~m} \times$ $0.25 \mathrm{~mm}$ i.d. fused silica capillary column (CP-Sil 88, Chrompack, Middelburg, The Netherlands). Identification of 18:1, 18:2, CLA, and 18:3 isomers and odd and branched-chain fatty acids was as described in Loor et al. (2004b). A butter reference standard (BCR 164; Commission of the European Communities, Community Bureau of Reference, Brussels, Belgium) was used to estimate correction factors for short-chain (4:0-10:0) fatty acids.

For fatty acid analysis ( 0.5 to $1 \mu \mathrm{L}$ of methyl esters in hexane injected at a 50:1 split ratio), the injector temperature was maintained at $250^{\circ} \mathrm{C}$ and the detector temperature was maintained at $255^{\circ} \mathrm{C}$. The oven tem- perature was held at $70^{\circ} \mathrm{C}$ for $1 \mathrm{~min}$, increased at $5^{\circ} \mathrm{C} /$ min to $100^{\circ} \mathrm{C}$ (held for $2 \mathrm{~min}$ ), then increased at $10^{\circ} \mathrm{C} /$ $\min$ to $175^{\circ} \mathrm{C}$ (held for $40 \mathrm{~min}$ ), and increased at $5^{\circ} \mathrm{C} /$ min to a final temperature of $225^{\circ} \mathrm{C}$ (held for $15 \mathrm{~min}$ ). Hydrogen was the carrier gas. Injector pressure was held constant at $158.6 \mathrm{kPa}$. For all samples, satisfactory separations of cis- and trans-18:1 and nonconjugated 18:2 isomers were obtained with a single chromatographic run (see Figure 1 in Loor et al., 2004b). Separation of CLA isomers in duodenal lipids was shown in Loor et al. (2005c). Diurnal profiles of trans10-18:1, trans11-18:1, cis9,trans11-18:2, trans10,cis12-18:2, trans11,cis15-18:2, and 18:0 in ruminal fluid were presented in Loor et al. (2004a). Intake and duodenal flows of fatty acids were presented in Loor et al. (2005c).

\section{Statistical Analyses}

Data for DMI, milk production and composition, fatty acids in plasma, ruminal fluid, and milk fat are reported as least squares means \pm SEM. Data were analyzed as a Latin square using the MIXED procedure of SAS (SAS Inst., Inc., Cary, NC). The statistical model included: cow, period, oil type, and residual error. Fixed effects included period and oil type. Cow was the random effect. Compound symmetry was the covariate structure used. Overall differences between treatment means were considered significant when $P \leq 0.05$.

\section{RESULTS}

\section{Production Variables and Blood Plasma Metabolites}

Average DMI and milk, fat, protein, and lactose concentration and yields did not differ in response to oil type (Table 1). In cows fed LO, triglyceride concentration in jugular plasma and jugular-mammary venous difference were higher compared with other treatments (Table 2). In contrast, feeding SO resulted in higher jugular concentrations of glucose. The jugular-mammary venous difference for insulin was greater with FO compared with other treatments. Across diets there appeared to be net production of leptin by the mammary gland (i.e., significantly negative jugular-mammary venous difference), in agreement with data on leptin expression in this tissue (Bonnet et al., 2002).

\section{Fatty Acid Composition of Ruminal Fluid}

Among 18:1 isomers, percentage of trans 10-18:1 was similar between FO and SO, but FO increased cis1118:1 to the largest extent (Table 3). Among 18:2 isomers, both FO and LO resulted in greater trans11,cis15-18:2 
Table 1. Dry matter intake and milk production and composition in Holstein cows fed a high-concentrate diet supplemented with fish oil (FO, $2.5 \%$ of DM), linseed oil (LO, 5\%), or sunflower oil (SO, 5\%).

\begin{tabular}{lccccc}
\hline & \multicolumn{3}{c}{ Supplement } & & \\
\cline { 2 - 5 } & FO & LO & SO & SEM & $P=$ \\
\hline DMI, kg/d & 17.1 & 17.2 & 19.3 & 2.4 & 0.78 \\
Milk, kg/d & 27.2 & 24.4 & 26.5 & 6.9 & 0.70 \\
Composition, \% & & & & & \\
Fat & 2.56 & 2.75 & 2.62 & 0.36 & 0.57 \\
Protein & 2.97 & 3.18 & 3.50 & 0.28 & 0.10 \\
Lactose & 4.77 & 4.71 & 4.68 & 0.10 & 0.72 \\
Yield, kg/d & & & & & 0.98 \\
Fat & 0.69 & 0.71 & 0.72 & 0.14 & 0.39 \\
Protein & 0.79 & 0.75 & 0.86 & 0.33 & 0.71 \\
Lactose & 1.30 & 1.14 & 1.26 & & \\
\hline
\end{tabular}

than did SO. There also were unique responses for individual oils. Feeding LO resulted in lower trans10-18:1 and in greater percentages of trans $13+14-18: 1$, trans 15 18:1, trans $16-18: 1$, cis 12-18:1, and cis $15-18: 1$. Feeding SO, however, resulted in higher percentages of cis 9 ,cis12-18:2, cis9,trans11-18:2, and trans10,cis1218:2, and decreased trans11,trans13-18:2. Feeding LO increased percentages of trans 9, cis $12-18: 2$, cis 9 ,trans13-18:2, trans 9 ,trans12-18:2, trans11,cis15-18:2, and trans $11, c i s 13+$ cis 9, cis 11-18:2, and decreased trans,trans-18:2. Feeding LO also resulted in greater percentages of cis9,cis 12, cis 15-18:3 and trans9,trans12,trans15-18:3. Dietary FO resulted in markedly lower 18:0, and increased percentages of cis11-18:1, trans8, cis13-18:2, trans9,cis11-18:2, cis10,trans 12 18:2, cis 11 ,trans $13-18: 2,20: 2,20: 3 n-3,20: 3 n-6,20: 4 n-$ $6,20: 5 n-3,21: 0,22: 1 n-9,22: 5 n-3$, and 22:6n-3.

\section{Plasma Fatty Acid Concentrations}

Despite differences in the amount of supplemental oil fed, concentration of total fatty acids in plasma did

Table 2. Plasma metabolites and hormones, and apparent mammary uptake in Holstein cows fed a highconcentrate diet supplemented with fish oil (FO, 2.5\% of DM), linseed oil (LO, 5\%), or sunflower oil (SO, $5 \%$ ).

\begin{tabular}{|c|c|c|c|c|c|}
\hline & \multicolumn{3}{|c|}{ Supplement } & \multirow[b]{2}{*}{ SEM } & \multirow[b]{2}{*}{$P=$} \\
\hline & FO & LO & SO & & \\
\hline \multicolumn{6}{|c|}{ Jugular concentration, $\mathrm{mmol} / \mathrm{L}$} \\
\hline Acetate & 0.463 & 0.596 & 0.413 & 0.103 & 0.49 \\
\hline BHBA & 0.434 & 0.475 & 0.398 & 0.058 & 0.66 \\
\hline Lactate & 1.189 & 0.739 & 1.237 & 0.307 & 0.51 \\
\hline Glucose & $3.55^{\mathrm{b}}$ & $3.62^{\mathrm{ab}}$ & $3.80^{\mathrm{a}}$ & 0.09 & 0.05 \\
\hline Total cholesterol & 6.23 & 6.78 & 7.64 & 1.14 & 0.57 \\
\hline Phospholipid & 3.14 & 3.17 & 3.48 & 0.46 & 0.78 \\
\hline Triglyceride & $0.100^{\mathrm{b}}$ & $0.154^{\mathrm{a}}$ & $0.133^{\mathrm{ab}}$ & 0.01 & 0.05 \\
\hline Free glycerol & 0.185 & 0.258 & 0.230 & 0.047 & 0.58 \\
\hline NEFA & 0.129 & 0.235 & 0.177 & 0.044 & 0.11 \\
\hline Urea & 4.59 & 4.29 & 4.28 & 0.43 & 0.84 \\
\hline Insulin, $\mu \mathrm{IU} / \mathrm{mL}$ & 22.1 & 16.6 & 22.7 & 2.0 & 0.12 \\
\hline Leptin, ng/mL & 6.22 & 6.15 & 6.28 & 1.78 & 0.99 \\
\hline \multicolumn{6}{|c|}{ Jugular-mammary venous difference } \\
\hline Acetate & 0.331 & 0.444 & 0.262 & 0.063 & 0.11 \\
\hline BHBA & 0.121 & 0.174 & 0.108 & 0.038 & 0.50 \\
\hline Lactate & 0.379 & 0.075 & 0.119 & 0.224 & 0.62 \\
\hline Glucose & 0.760 & 0.906 & 0.859 & 0.229 & 0.56 \\
\hline Total cholesterol & -1.24 & -0.23 & -0.17 & 0.76 & 0.57 \\
\hline Phospholipid & -0.36 & -0.12 & -0.12 & 0.25 & 0.75 \\
\hline Triglyceride & $0.062^{\mathrm{b}}$ & $0.093^{\mathrm{a}}$ & $0.066^{\mathrm{b}}$ & 0.010 & 0.05 \\
\hline Free glycerol & 0.025 & 0.064 & 0.045 & 0.068 & 0.92 \\
\hline NEFA & -0.053 & -0.179 & -0.105 & 0.064 & 0.45 \\
\hline Urea & $-0.045^{\mathrm{b}}$ & $0.080^{\mathrm{a}}$ & $-0.035^{\mathrm{b}}$ & 0.038 & 0.05 \\
\hline Insulin, $\mu \mathrm{IU} / \mathrm{mL}$ & $5.85^{\mathrm{a}}$ & $-7.45^{\mathrm{b}}$ & $-4.62^{\mathrm{b}}$ & 3.96 & 0.05 \\
\hline Leptin, ng/mL & -0.55 & -0.17 & -0.31 & 0.28 & 0.52 \\
\hline
\end{tabular}

${ }^{\mathrm{a}, \mathrm{b}}$ Means with unlike superscripts differ significantly $(P<0.05)$. 
Table 3. Percentage of selected fatty acids in ruminal fluid from Holstein cows fed a high-concentrate diet supplemented with fish oil (FO, 2.5\% of DM), linseed oil (LO, 5\%), or sunflower oil (SO, 5\%).

\begin{tabular}{|c|c|c|c|c|c|}
\hline \multirow[b]{2}{*}{ Fatty acid } & \multicolumn{3}{|c|}{ Supplement } & \multirow[b]{2}{*}{ SEM } & \multirow[b]{2}{*}{$P=$} \\
\hline & FO & LO & $\mathrm{SO}$ & & \\
\hline & \multicolumn{3}{|c|}{$\longrightarrow \mathrm{g} / 100 \mathrm{~g}$ of total fatty acids } & & \\
\hline $16: 0$ & $24.5^{\mathrm{a}}$ & $13.9^{\mathrm{c}}$ & $17.5^{\mathrm{b}}$ & 0.66 & 0.001 \\
\hline $18: 0$ & $11.9^{\mathrm{b}}$ & $25.6^{\mathrm{a}}$ & $24.3^{\mathrm{a}}$ & 6.4 & 0.001 \\
\hline \multicolumn{6}{|l|}{ 18:1 trans } \\
\hline 4 & 0.18 & 0.16 & 0.17 & 0.03 & 0.66 \\
\hline 5 & 0.10 & 0.11 & 0.12 & 0.01 & 0.69 \\
\hline $6+7+8$ & 0.76 & 0.99 & 0.99 & 0.11 & 0.09 \\
\hline 9 & 0.49 & 0.58 & 0.66 & 0.04 & 0.13 \\
\hline 10 & $8.25^{\mathrm{a}}$ & $3.06^{\mathrm{b}}$ & $8.35^{\mathrm{a}}$ & 3.11 & 0.05 \\
\hline 11 & 9.20 & 11.4 & 11.0 & 2.00 & 0.30 \\
\hline 12 & 1.14 & 1.19 & 1.19 & 0.08 & 0.86 \\
\hline $13+14$ & $1.46^{\mathrm{c}}$ & $4.16^{\mathrm{a}}$ & $1.80^{\mathrm{b}}$ & 0.19 & 0.001 \\
\hline 15 & $0.68^{\mathrm{b}}$ & $1.85^{\mathrm{a}}$ & $0.78^{b}$ & 0.08 & 0.001 \\
\hline 16 & $0.37^{b}$ & $1.15^{\mathrm{a}}$ & $0.52^{\mathrm{b}}$ & 0.08 & 0.001 \\
\hline \multicolumn{6}{|l|}{$18: 1 \mathrm{cis}$} \\
\hline 9 & 5.20 & 5.49 & 5.63 & 0.87 & 0.74 \\
\hline 11 & $2.19^{\mathrm{a}}$ & $0.97^{\mathrm{b}}$ & $1.13^{\mathrm{b}}$ & 0.29 & 0.001 \\
\hline 12 & $0.32^{\mathrm{c}}$ & $1.04^{\mathrm{a}}$ & $0.87^{\mathrm{b}}$ & 0.10 & 0.01 \\
\hline 13 & 0.15 & 0.20 & 0.11 & 0.01 & 0.07 \\
\hline 15 & $0.41^{\mathrm{b}}$ & $1.58^{\mathrm{a}}$ & $0.19^{b}$ & 0.20 & 0.001 \\
\hline \multicolumn{6}{|l|}{ Nonconjugated 18:2 } \\
\hline cis 9, cis 12 & $3.65^{\mathrm{b}}$ & $3.36^{\mathrm{b}}$ & $9.48^{\mathrm{a}}$ & 1.69 & 0.05 \\
\hline cis 9, trans 12 & $0.14^{\mathrm{b}}$ & $0.22^{\mathrm{a}}$ & $0.13^{\mathrm{b}}$ & 0.01 & 0.001 \\
\hline trans $8, \operatorname{cis} 13$ & $0.57^{\mathrm{a}}$ & $0.22^{\mathrm{b}}$ & $0.32^{\mathrm{b}}$ & 0.10 & 0.04 \\
\hline trans 9, cis 12 & $0.19^{b}$ & $0.35^{\mathrm{a}}$ & $0.16^{\mathrm{b}}$ & 0.07 & 0.01 \\
\hline cis 9, trans 13 & $0.20^{\mathrm{b}}$ & $0.28^{\mathrm{a}}$ & $0.12^{\mathrm{c}}$ & 0.03 & 0.001 \\
\hline $\operatorname{trans} 9, \operatorname{trans} 12$ & $0.22^{\mathrm{b}}$ & $0.39^{\mathrm{a}}$ & $0.10^{\mathrm{b}}$ & 0.13 & 0.02 \\
\hline trans 11, cis 15 & $2.93^{\mathrm{b}}$ & $8.56^{\mathrm{a}}$ & $0.95^{\mathrm{c}}$ & 1.29 & 0.001 \\
\hline \multicolumn{6}{|l|}{ Conjugated 18:2 } \\
\hline cis 9, trans 11 & $0.73^{\mathrm{b}}$ & $0.70^{\mathrm{b}}$ & $1.46^{\mathrm{a}}$ & 0.13 & 0.04 \\
\hline $\operatorname{trans} 9$, cis 11 & $0.07^{\mathrm{a}}$ & $0.01^{\mathrm{b}}$ & $0.01^{b}$ & 0.02 & 0.004 \\
\hline trans8,cis 10 & 0.08 & 0.05 & 0.05 & 0.01 & 0.16 \\
\hline trans 10, cis 12 & $0.07^{\mathrm{b}}$ & $0.06^{\mathrm{b}}$ & $0.23^{\mathrm{a}}$ & 0.04 & 0.01 \\
\hline cis 10, trans 12 & $0.04^{\mathrm{a}}$ & $0.002^{\mathrm{b}}$ & $0.02^{\mathrm{b}}$ & 0.01 & 0.05 \\
\hline cis 11, trans 13 & $0.05^{\mathrm{a}}$ & $0.02^{\mathrm{b}}$ & $0.01^{\mathrm{b}}$ & 0.01 & 0.05 \\
\hline trans $11, c i s 13+\operatorname{cis} 9, \operatorname{cis} 11$ & $0.08^{\mathrm{b}}$ & $0.15^{\mathrm{a}}$ & $0.09^{b}$ & 0.01 & 0.05 \\
\hline trans 11, trans 13 & $0.18^{\mathrm{a}}$ & $0.23^{\mathrm{a}}$ & $0.09^{b}$ & 0.03 & 0.02 \\
\hline trans,trans & $0.33^{\mathrm{a}}$ & $0.18^{\mathrm{b}}$ & $0.30^{\mathrm{a}}$ & 0.03 & 0.05 \\
\hline \multicolumn{6}{|l|}{$18: 3$} \\
\hline cis 9, cis $12, c i s 15$ & $1.20^{\mathrm{b}}$ & $2.46^{\mathrm{a}}$ & $0.88^{\mathrm{c}}$ & 0.25 & 0.04 \\
\hline trans 9, trans 12, trans 15 & $0.24^{\mathrm{b}}$ & $0.56^{\mathrm{a}}$ & $0.09^{\mathrm{c}}$ & 0.08 & 0.001 \\
\hline $20: 2$ & $0.13^{\mathrm{a}}$ & $0.07^{\mathrm{b}}$ & $0.07^{\mathrm{b}}$ & 0.01 & 0.04 \\
\hline $20: 3 n-3$ & $0.31^{\mathrm{a}}$ & $0.02^{\mathrm{b}}$ & $0.05^{b}$ & 0.05 & 0.01 \\
\hline $20: 3 n-6$ & $0.08^{\mathrm{a}}$ & $0.004^{\mathrm{b}}$ & $0.02^{\mathrm{b}}$ & 0.02 & 0.02 \\
\hline $20: 4 n-6$ & $0.06^{\mathrm{a}}$ & $0.001^{\mathrm{b}}$ & $0.01^{\mathrm{b}}$ & 0.01 & 0.03 \\
\hline $20: 5 n-3$ & $1.77^{\mathrm{a}}$ & $0.01^{\mathrm{b}}$ & $0.10^{\mathrm{b}}$ & 0.20 & 0.001 \\
\hline 21:0 & $0.32^{\mathrm{a}}$ & $0.05^{\mathrm{b}}$ & $0.08^{b}$ & 0.06 & 0.01 \\
\hline $22: 0$ & $0.54^{\mathrm{b}}$ & $0.30^{c}$ & $0.59^{\mathrm{a}}$ & 0.02 & 0.001 \\
\hline $22: 1 n-9$ & $0.15^{\mathrm{a}}$ & $0.05^{\mathrm{b}}$ & $0.06^{\mathrm{b}}$ & 0.01 & 0.001 \\
\hline $22: 5 n-3$ & $0.54^{\mathrm{a}}$ & $0.01^{\mathrm{b}}$ & $0.07^{\mathrm{b}}$ & 0.09 & 0.001 \\
\hline $22: 6 n-3$ & $0.86^{\mathrm{a}}$ & $0.04^{\mathrm{b}}$ & $0.12^{\mathrm{b}}$ & 0.09 & 0.001 \\
\hline Total, ${ }^{1} \mathrm{mg} / \mathrm{g}$ of DM & 29.9 & 38.6 & 34.9 & 3.28 & 0.10 \\
\hline
\end{tabular}

${ }^{\mathrm{a}, \mathrm{b}, \mathrm{c}}$ Means with unlike superscripts differ significantly $(P<0.05)$.

${ }^{1}$ Sum of fatty acids with 12 - to 24 -carbons including 18:1, 18:2, and 18:3 isomers.

not differ due to treatments (Table 4). Among oils, feeding $\mathrm{FO}$ resulted in greater concentrations of 14:0, 16:0, most odd- and branched-chain fatty acids, and nearly all 20-carbon, 22-carbon, and 24-carbon fatty acids. In contrast, FO decreased concentration of 18:0 dramatically compared with LO and SO.

\section{Plasma 18:1 Isomer Concentrations}

Total trans-18:1 in plasma was greater with FO than other treatments (Table 5). Among individual isomers, the higher concentration of trans 11-18:1 primarily, but also trans 10-18:1, accounted for the greater response 
Table 4. Concentration of 12- to 22-carbon fatty acids (not including 18:1, 18:2, or 18:3 isomers) in jugular blood plasma from Holstein cows fed a high-concentrate diet supplemented with fish oil (FO, 2.5\% of DM), linseed oil (LO, 5\%), or sunflower oil (SO, 5\%).

\begin{tabular}{|c|c|c|c|c|c|}
\hline \multirow[b]{2}{*}{ Fatty acid } & \multicolumn{3}{|c|}{ Supplement } & \multirow[b]{2}{*}{ SEM } & \multirow[b]{2}{*}{$P=$} \\
\hline & $\mathrm{FO}$ & $\mathrm{LO}$ & SO & & \\
\hline & & $\mu \mathrm{g} / \mathrm{mL}-$ & - & & \\
\hline $12: 0$ & 1.95 & 1.30 & 1.24 & 0.32 & 0.31 \\
\hline $14: 0$ & $18.6^{\mathrm{a}}$ & $12.0^{\mathrm{b}}$ & $12.5^{\mathrm{b}}$ & 1.5 & 0.05 \\
\hline iso-14:0 & 1.06 & 1.18 & 1.31 & 0.08 & 0.24 \\
\hline cis9-14:1 & 1.22 & 1.04 & 1.23 & 0.17 & 0.69 \\
\hline $15: 0$ & $24.1^{\mathrm{a}}$ & $12.8^{\mathrm{b}}$ & $13.6^{\mathrm{b}}$ & 3.1 & 0.04 \\
\hline cis 10-15:1 & 3.18 & 1.88 & 2.59 & 0.97 & 0.67 \\
\hline iso-15:0 & $10.5^{\mathrm{a}}$ & $6.60^{\mathrm{b}}$ & $6.43^{\mathrm{b}}$ & 1.37 & 0.05 \\
\hline anteiso-15:0 & 12.7 & 13.7 & 11.5 & 2.3 & 0.62 \\
\hline $16: 0$ & $332.4^{\mathrm{a}}$ & $197.8^{\mathrm{b}}$ & $221.8^{\mathrm{b}}$ & 48.0 & 0.02 \\
\hline iso- $16: 0$ & $10.1^{\mathrm{a}}$ & $5.66^{\mathrm{b}}$ & $6.78^{\mathrm{b}}$ & 1.38 & 0.05 \\
\hline $\operatorname{trans} 9-16: 1+i s o-17: 0$ & $18.5^{\mathrm{a}}$ & $8.45^{\mathrm{b}}$ & $12.2^{\mathrm{b}}$ & 3.86 & 0.02 \\
\hline cis $9-16: 1$ & $67.4^{\mathrm{a}}$ & $25.7^{\mathrm{b}}$ & $19.7^{\mathrm{b}}$ & 8.7 & 0.03 \\
\hline $17: 0$ & $25.4^{\mathrm{a}}$ & $14.3^{\mathrm{b}}$ & $17.7^{\mathrm{b}}$ & 3.7 & 0.001 \\
\hline $\operatorname{cis} 10-17: 1$ & 4.40 & 5.00 & 3.66 & 0.78 & 0.10 \\
\hline $18: 0$ & $228.3^{\mathrm{b}}$ & $354.5^{\mathrm{a}}$ & $384.9^{\mathrm{a}}$ & 32.1 & 0.05 \\
\hline 19:0 & 5.13 & 3.48 & 4.23 & 1.14 & 0.63 \\
\hline $20: 0$ & $4.89^{\mathrm{a}}$ & $3.72^{\mathrm{b}}$ & $2.99^{\mathrm{b}}$ & 0.54 & 0.05 \\
\hline $\operatorname{cis} 11-20: 1$ & $4.44^{\mathrm{a}}$ & $2.98^{\mathrm{b}}$ & $3.06^{\mathrm{b}}$ & 0.33 & 0.05 \\
\hline $20: 2$ & $7.32^{\mathrm{a}}$ & $4.29^{\mathrm{b}}$ & $4.71^{\mathrm{b}}$ & 0.58 & 0.05 \\
\hline $20: 3 n-3$ & $5.71^{\mathrm{a}}$ & $0.82^{\mathrm{b}}$ & $0.98^{\mathrm{b}}$ & 1.20 & 0.05 \\
\hline $20: 3 n-6$ & 24.4 & 22.2 & 23.1 & 4.9 & 0.95 \\
\hline $20: 4 n-6$ & $57.6^{\mathrm{a}}$ & $17.4^{\mathrm{b}}$ & $26.4^{\mathrm{b}}$ & 8.5 & 0.05 \\
\hline $20: 5 n-3$ & $352.1^{\mathrm{a}}$ & $42.2^{\mathrm{b}}$ & $77.2^{\mathrm{b}}$ & 88.0 & 0.05 \\
\hline $22: 0$ & $4.26^{\mathrm{a}}$ & $2.51^{\mathrm{b}}$ & $2.27^{\mathrm{b}}$ & 0.49 & 0.05 \\
\hline $22: 1 n-9$ & 32.2 & 13.6 & 9.02 & 20.3 & 0.72 \\
\hline $22: 2$ & 1.38 & 0.64 & 1.03 & 0.41 & 0.50 \\
\hline $22: 4 n-6$ & 1.24 & 1.92 & 1.46 & 0.30 & 0.37 \\
\hline $22: 5 n-3$ & $38.0^{\mathrm{a}}$ & $24.4^{\mathrm{b}}$ & $32.0^{\mathrm{a}}$ & 6.3 & 0.05 \\
\hline $22: 6 n-3$ & $30.8^{\mathrm{a}}$ & $8.37^{\mathrm{b}}$ & $15.3^{\mathrm{b}}$ & 5.54 & 0.002 \\
\hline $24: 0$ & $6.92^{\mathrm{a}}$ & $2.10^{\mathrm{b}}$ & $2.62^{\mathrm{b}}$ & 1.30 & 0.05 \\
\hline $24: 1 n-9$ & 0.68 & 0.60 & 0.48 & 0.17 & 0.71 \\
\hline Total $^{1}$ & 2823 & 2630 & 2914 & 415 & 0.49 \\
\hline
\end{tabular}

${ }^{\mathrm{a}, \mathrm{b}}$ Means with unlike superscripts differ significantly $(P<0.05)$.

${ }^{1}$ Total $=$ sum of $12: 0$ to $24: 1 n-9$ including $18: 1,18: 2$, and 18:3 isomers.

with FO. Feeding SO also increased trans10-18:1 concentration compared with LO. In contrast, LO supplementation resulted in greater concentrations of trans $13+14-18: 1$, trans $15-18: 1$, and trans $16-18: 1$ compared with FO or LO. Contrary to results for trans18:1, LO increased total cis-18:1 to the largest extent, and this response was due primarily to greater cis918:1 and cis 15-18:1. FO resulted in greater cis11-18:1 compared with LO or SO.

\section{Plasma 18:2 and 18:3 Isomer Concentrations}

Total concentration of 18:3 isomers was greater with LO, which resulted from higher cis9,cis 12 ,cis 15-18:3 and trans9,trans 12 ,trans 15-18:3 (Table 6). Interestingly, concentration of total conjugated 18:2 isomers was greater when FO was fed compared with SO and intermediate with LO. Among isomers that increased with FO were trans9,cis11-18:2, cis10,trans12-18:2, and trans,trans-18:2, and nonconjugated trans11,cis1518:2. Circulating concentration of cis9,trans 11-18:2 was higher when LO was fed compared with $\mathrm{FO}$, and intermediate with SO. Feeding LO also increased concentration of trans11,cis13- + cis9,cis11-18:2 compared with other treatments. Feeding SO resulted in greater concentrations of trans 10,cis 12-18:2 and lower trans 8 ,cis 10 compared with FO and LO. Concentration of total nonconjugated 18:2 isomers was greater with SO than other treatments, primarily due to a substantial increase in cis9,cis12-18:2. Among other isomers, cis9,trans1218:2, cis9,trans $13-18: 2$, and trans 11 ,cis $15-18: 2$ were greater in response to LO compared with other treatments.

\section{Percentage and Yield of Selected Fatty Acids in Milk}

Cows fed FO had higher percentages of 8:0, 10:0, cis $9-10: 1,12: 0,13: 0,14: 0,15: 0,16: 0$, most odd- and 
Table 5. Concentration of 18:1 isomers in blood plasma from Holstein cows fed a high-concentrate diet supplemented with fish oil (FO, $2.5 \%$ of DM), linseed oil (LO, 5\%), or sunflower oil (SO, 5\%).

\begin{tabular}{|c|c|c|c|c|c|}
\hline \multirow[b]{2}{*}{ Isomer } & \multicolumn{3}{|c|}{ Supplement } & \multirow[b]{2}{*}{ SEM } & \multirow[b]{2}{*}{$P=$} \\
\hline & FO & $\mathrm{LO}$ & $\mathrm{SO}$ & & \\
\hline & & $\mu \mathrm{g} / \mathrm{mL}$ & - & & \\
\hline \multicolumn{6}{|l|}{ 18:1 trans } \\
\hline 4 & 3.55 & 3.69 & 3.72 & 0.51 & 0.59 \\
\hline 5 & 2.51 & 3.02 & 2.90 & 0.74 & 0.88 \\
\hline $6+7+8$ & 5.98 & 5.84 & 6.13 & 1.27 & 0.98 \\
\hline 9 & 7.07 & 5.42 & 6.14 & 1.10 & 0.49 \\
\hline 10 & $41.1^{\mathrm{a}}$ & $11.3^{\mathrm{b}}$ & $31.1^{\mathrm{a}}$ & 10.7 & 0.05 \\
\hline 11 & $115.6^{\mathrm{a}}$ & $64.5^{\mathrm{b}}$ & $58.4^{\mathrm{b}}$ & 15.8 & 0.05 \\
\hline 12 & 14.3 & 16.1 & 16.4 & 3.0 & 0.49 \\
\hline $13+14$ & $16.8^{\mathrm{b}}$ & $41.1^{\mathrm{a}}$ & $18.0^{\mathrm{b}}$ & 7.3 & 0.05 \\
\hline 15 & $5.00^{\mathrm{b}}$ & $14.4^{\mathrm{a}}$ & $5.53^{\mathrm{b}}$ & 2.29 & 0.05 \\
\hline 16 & $3.15^{\mathrm{b}}$ & $11.7^{\mathrm{a}}$ & $5.22^{\mathrm{b}}$ & 1.31 & 0.04 \\
\hline Total & $210.1^{\mathrm{a}}$ & $162.6^{\mathrm{b}}$ & $148.2^{\mathrm{b}}$ & 23.1 & 0.04 \\
\hline \multicolumn{6}{|l|}{$18: 1 \mathrm{cis}$} \\
\hline 9 & $90.2^{\mathrm{b}}$ & $152.6^{\mathrm{a}}$ & $110.8^{\mathrm{b}}$ & 14.8 & 0.05 \\
\hline 11 & $30.3^{\mathrm{a}}$ & $14.3^{\mathrm{b}}$ & $14.8^{\mathrm{b}}$ & 3.6 & 0.01 \\
\hline 12 & 16.6 & 35.2 & 25.5 & 7.9 & 0.19 \\
\hline 13 & 2.85 & 4.56 & 3.19 & 0.72 & 0.19 \\
\hline 15 & $4.15^{\mathrm{b}}$ & $14.0^{\mathrm{a}}$ & $3.06^{\mathrm{b}}$ & 3.22 & 0.04 \\
\hline Total & $149.0^{\mathrm{b}}$ & $235.0^{\mathrm{a}}$ & $162.9^{\mathrm{b}}$ & 20.2 & 0.05 \\
\hline
\end{tabular}

${ }^{\mathrm{a}, \mathrm{b}}$ Means with unlike superscripts differ significantly $(P<0.05)$.

branched-chain fatty acids, all 20-carbon fatty acids, and most 22-carbon fatty acids (Table 7). However, similar to ruminal fluid and plasma, the percentage and yield of 18:0 when FO was fed were much lower com- pared with LO and SO. Despite greater percentages in milk in cows fed FO, yields of saturated fatty acids with 8 - to 14-carbons did not differ across treatments. Yields of total 20-carbon and 20:5n-3, 22:5n-3, and 22:6n-3

Table 6. Concentration of 18:2 and 18:3 isomers in blood plasma from Holstein cows fed a high-concentrate diet supplemented with fish oil (FO, $2.5 \%$ of DM), linseed oil (LO, 5\%), or sunflower oil (SO, 5\%).

\begin{tabular}{|c|c|c|c|c|c|}
\hline \multirow[b]{2}{*}{ Isomer } & \multicolumn{3}{|c|}{ Supplement } & \multirow[b]{2}{*}{ SEM } & \multirow[b]{2}{*}{$P=$} \\
\hline & FO & $\mathrm{LO}$ & SO & & \\
\hline & - & $-\mu \mathrm{g} / \mathrm{mL}$ & - & & \\
\hline \multicolumn{6}{|l|}{$18: 3$} \\
\hline cis9,cis $12, c i s 15$ & $150.0^{\mathrm{b}}$ & $353.1^{\mathrm{a}}$ & $140.7^{\mathrm{b}}$ & 41.4 & 0.01 \\
\hline trans 9, trans $12, \operatorname{trans} 15$ & $5.10^{\mathrm{b}}$ & $16.0^{\mathrm{a}}$ & $3.54^{\mathrm{b}}$ & 2.23 & 0.05 \\
\hline Total & $154.9^{\mathrm{b}}$ & $369.1^{\mathrm{a}}$ & $144.3^{\mathrm{b}}$ & 44.3 & 0.01 \\
\hline \multicolumn{6}{|l|}{ 18:2 conjugated } \\
\hline cis 9, trans 11 & $6.90^{\mathrm{b}}$ & $10.4^{\mathrm{a}}$ & $8.36^{\mathrm{ab}}$ & 0.76 & 0.05 \\
\hline $\operatorname{trans} 9, \operatorname{cis} 11$ & $3.23^{\mathrm{a}}$ & $0.00^{\mathrm{b}}$ & $0.54^{\mathrm{b}}$ & 0.66 & 0.05 \\
\hline trans 8 ,cis 10 & $1.28^{\mathrm{a}}$ & $1.40^{\mathrm{a}}$ & $0.82^{\mathrm{b}}$ & 0.13 & 0.05 \\
\hline trans 10, cis 12 & $2.42^{\mathrm{b}}$ & $1.87^{\mathrm{b}}$ & $3.26^{\mathrm{a}}$ & 0.30 & 0.05 \\
\hline cis 10, trans 12 & $5.57^{\mathrm{a}}$ & $0.39^{\mathrm{c}}$ & $1.10^{\mathrm{b}}$ & 0.30 & 0.01 \\
\hline cis 11, trans 13 & 0.00 & 0.50 & 0.56 & 0.33 & 0.43 \\
\hline trans 11, cis $13+\operatorname{cis} 9$, cis 11 & $0.00^{\mathrm{b}}$ & $0.86^{\mathrm{a}}$ & $0.00^{\mathrm{b}}$ & 0.29 & 0.05 \\
\hline trans 11, trans 13 & 2.51 & 4.61 & 2.42 & 1.12 & 0.38 \\
\hline trans,trans & $12.4^{\mathrm{a}}$ & $7.31^{\mathrm{b}}$ & $5.26^{\mathrm{b}}$ & 0.84 & 0.01 \\
\hline Total & $34.3^{\mathrm{a}}$ & $27.3^{\mathrm{ab}}$ & $22.3^{\mathrm{b}}$ & 2.43 & 0.05 \\
\hline \multicolumn{6}{|l|}{ 18:2 nonconjugated } \\
\hline $\operatorname{cis} 9, \operatorname{cis} 12$ & $900.4^{\mathrm{b}}$ & $969.1^{\mathrm{b}}$ & $1483^{\mathrm{a}}$ & 148 & 0.02 \\
\hline cis 9, trans 12 & $2.38^{\mathrm{b}}$ & $4.91^{\mathrm{a}}$ & $2.39^{\mathrm{b}}$ & 0.59 & 0.05 \\
\hline $\operatorname{trans} 8, \operatorname{cis} 13$ & 7.46 & 9.33 & 8.47 & 1.32 & 0.64 \\
\hline trans 9, cis 12 & 6.81 & 4.60 & 5.70 & 1.00 & 0.11 \\
\hline cis 9, trans 13 & $4.06^{\mathrm{b}}$ & $7.80^{\mathrm{a}}$ & $4.65^{\mathrm{b}}$ & 1.00 & 0.05 \\
\hline trans 9, trans 12 & 1.71 & 2.60 & 1.72 & 0.62 & 0.47 \\
\hline trans 11, cis 15 & $13.5^{\mathrm{b}}$ & $24.1^{\mathrm{a}}$ & $4.69^{\mathrm{c}}$ & 2.77 & 0.02 \\
\hline Total & $936.4^{\mathrm{b}}$ & $1022^{\mathrm{b}}$ & $1511^{\mathrm{a}}$ & 154.9 & 0.03 \\
\hline
\end{tabular}

${ }^{\mathrm{a}, \mathrm{b}, \mathrm{c}}$ Means with unlike superscripts differ significantly $(P<0.05)$. 
Table 7. Percentage of 4- to 24-carbon (not including 18:1, 18:2, or 18:3 isomers) fatty acids and yield of selected fatty acids in milk from Holstein cows fed a high-concentrate diet supplemented with fish oil (FO, $2.5 \%$ of $\mathrm{DM})$, linseed oil (LO, $5 \%$ ), or sunflower oil (SO, 5\%).

\begin{tabular}{|c|c|c|c|c|c|}
\hline \multirow[b]{2}{*}{ Fatty acid } & \multicolumn{3}{|c|}{ Supplement } & \multirow[b]{2}{*}{ SEM } & \multirow[b]{2}{*}{$P=$} \\
\hline & FO & LO & $\mathrm{SO}$ & & \\
\hline & \multicolumn{3}{|c|}{$\longrightarrow \mathrm{g} / 100 \mathrm{~g}$ of total fatty acids } & & \\
\hline $4: 0$ & 2.99 & 2.27 & 2.21 & 0.48 & 0.51 \\
\hline $6: 0$ & 1.66 & 1.17 & 1.14 & 0.34 & 0.36 \\
\hline $8: 0$ & $1.10^{\mathrm{a}}$ & $0.69^{b}$ & $0.64^{\mathrm{b}}$ & 0.10 & 0.05 \\
\hline 10:0 & $2.81^{\mathrm{a}}$ & $1.61^{\mathrm{b}}$ & $1.54^{\mathrm{b}}$ & 0.39 & 0.05 \\
\hline cis $9-10: 1$ & $0.22^{\mathrm{a}}$ & $0.15^{\mathrm{b}}$ & $0.13^{\mathrm{b}}$ & 0.03 & 0.05 \\
\hline $11: 0$ & 0.05 & 0.02 & 0.02 & 0.01 & 0.10 \\
\hline $12: 0$ & $3.43^{\mathrm{a}}$ & $1.98^{\mathrm{b}}$ & $2.03^{\mathrm{b}}$ & 0.36 & 0.05 \\
\hline $13: 0$ & $0.18^{\mathrm{a}}$ & $0.12^{\mathrm{b}}$ & $0.12^{\mathrm{b}}$ & 0.02 & 0.05 \\
\hline $14: 0$ & $12.5^{\mathrm{a}}$ & $8.46^{\mathrm{b}}$ & $8.99^{\mathrm{b}}$ & 1.02 & 0.05 \\
\hline $8: 0-14: 0$ & $19.8^{\mathrm{a}}$ & $12.7^{\mathrm{b}}$ & $13.2^{\mathrm{b}}$ & 1.78 & 0.05 \\
\hline iso-14:0 & 0.09 & 0.07 & 0.08 & 0.01 & 0.22 \\
\hline cis $9-14: 1$ & 0.99 & 0.76 & 0.86 & 0.14 & 0.59 \\
\hline $15: 0$ & $1.29^{\mathrm{a}}$ & $0.84^{\mathrm{b}}$ & $0.87^{\mathrm{b}}$ & 0.07 & 0.01 \\
\hline cis $10-15: 1$ & 0.02 & 0.02 & 0.02 & 0.01 & 0.96 \\
\hline iso-15:0 & $0.32^{\mathrm{a}}$ & $0.16^{\mathrm{b}}$ & $0.19^{b}$ & 0.04 & 0.05 \\
\hline anteiso-15:0 & $0.76^{\mathrm{a}}$ & $0.46^{\mathrm{b}}$ & $0.51^{b}$ & 0.06 & 0.04 \\
\hline $16: 0$ & $27.5^{\mathrm{a}}$ & $20.7^{\mathrm{b}}$ & $21.2^{\mathrm{b}}$ & 2.0 & 0.05 \\
\hline iso- $16: 0$ & $0.33^{\mathrm{a}}$ & $0.20^{\mathrm{b}}$ & $0.26^{\mathrm{b}}$ & 0.03 & 0.05 \\
\hline trans $9-16: 1+$ iso- $17: 0$ & $0.83^{\mathrm{a}}$ & $0.43^{\mathrm{b}}$ & $0.67^{\mathrm{b}}$ & 0.07 & 0.04 \\
\hline cis $9-16: 1$ & $2.00^{\mathrm{a}}$ & $1.18^{\mathrm{b}}$ & $1.41^{\mathrm{b}}$ & 0.23 & 0.05 \\
\hline $17: 0$ & $0.68^{\mathrm{a}}$ & $0.50^{\mathrm{b}}$ & $0.49^{\mathrm{b}}$ & 0.04 & 0.02 \\
\hline cis $10-17: 1$ & 0.25 & 0.20 & 0.19 & 0.03 & 0.43 \\
\hline $18: 0$ & $3.49^{\mathrm{b}}$ & $10.1^{\mathrm{a}}$ & $11.2^{\mathrm{a}}$ & 1.50 & 0.03 \\
\hline 19:0 & 0.15 & 0.16 & 0.16 & 0.03 & 0.98 \\
\hline $20: 0$ & 0.21 & 0.15 & 0.19 & 0.03 & 0.47 \\
\hline cis $11-20: 1$ & $0.54^{\mathrm{a}}$ & $0.14^{\mathrm{b}}$ & $0.18^{\mathrm{b}}$ & 0.04 & 0.01 \\
\hline $20: 2$ & 0.10 & 0.06 & 0.08 & 0.02 & 0.45 \\
\hline $20: 3 n-3$ & $0.45^{\mathrm{a}}$ & $0.02^{\mathrm{b}}$ & $0.02^{\mathrm{b}}$ & 0.10 & 0.05 \\
\hline $20: 3 n-6$ & $0.11^{\mathrm{a}}$ & $0.04^{\mathrm{b}}$ & $0.03^{\mathrm{b}}$ & 0.01 & 0.05 \\
\hline $20: 4 n-6$ & $0.14^{\mathrm{a}}$ & $0.07^{\mathrm{b}}$ & $0.05^{\mathrm{b}}$ & 0.01 & 0.05 \\
\hline $20: 5 n-3$ & $0.48^{\mathrm{a}}$ & $0.03^{\mathrm{b}}$ & $0.07^{\mathrm{b}}$ & 0.08 & 0.03 \\
\hline $21: 0$ & $0.14^{\mathrm{a}}$ & $0.05^{\mathrm{b}}$ & $0.06^{\mathrm{b}}$ & 0.02 & 0.05 \\
\hline $22: 0$ & $0.10^{\mathrm{a}}$ & $0.05^{\mathrm{c}}$ & $0.08^{b}$ & 0.001 & 0.02 \\
\hline $22: 1 n-9$ & $0.05^{\mathrm{a}}$ & $0.01^{\mathrm{b}}$ & $0.02^{\mathrm{b}}$ & 0.007 & 0.01 \\
\hline $22: 2$ & 0.01 & 0.01 & 0.01 & 0.004 & 0.45 \\
\hline $22: 5 n-3$ & $0.39^{\mathrm{a}}$ & $0.12^{\mathrm{b}}$ & $0.15^{\mathrm{b}}$ & 0.07 & 0.05 \\
\hline $22: 6 n-3$ & $0.19^{\mathrm{a}}$ & $0.04^{\mathrm{b}}$ & $0.06^{\mathrm{b}}$ & 0.03 & 0.05 \\
\hline $24: 0$ & 0.04 & 0.06 & 0.05 & 0.01 & 0.54 \\
\hline $24: 1 n-9$ & 0.02 & 0.01 & 0.02 & 0.003 & 0.22 \\
\hline \multicolumn{6}{|l|}{ Yield, g/d } \\
\hline $8: 0-14: 0$ & 126.0 & 88.5 & 99.1 & 37.6 & 0.21 \\
\hline Total odd + branched-chain & $30.2^{\mathrm{a}}$ & $19.6^{\mathrm{b}}$ & $21.6^{\mathrm{b}}$ & 5.9 & 0.01 \\
\hline $16: 0$ & 173.1 & 130.2 & 136.5 & 49.1 & 0.36 \\
\hline 18:0 & $22.3^{\mathrm{b}}$ & $70.0^{\mathrm{a}}$ & $78.0^{\mathrm{a}}$ & 19.5 & 0.05 \\
\hline Total 20-carbon & $17.2^{\mathrm{a}}$ & $4.36^{\mathrm{b}}$ & $4.98^{\mathrm{b}}$ & 2.61 & 0.01 \\
\hline $20: 5 n-3$ & $2.81^{\mathrm{a}}$ & $0.25^{\mathrm{b}}$ & $0.27^{\mathrm{b}}$ & 0.43 & 0.01 \\
\hline $22: 5 n-3$ & $2.14^{\mathrm{a}}$ & $0.63^{\mathrm{b}}$ & $0.72^{\mathrm{b}}$ & 0.27 & 0.02 \\
\hline $22: 6 n-3$ & $0.95^{\mathrm{a}}$ & $0.19^{\mathrm{b}}$ & $0.29^{b}$ & 0.15 & 0.05 \\
\hline
\end{tabular}

${ }^{\mathrm{a}, \mathrm{b}, \mathrm{c}}$ Means with unlike superscripts differ significantly $(P<0.05)$.

were greater in response to FO compared with other treatments, in agreement with their higher percentage in milk fat.

\section{Percentage and Yield of 18:1 Isomers}

Percentage and yield of total trans-18:1 isomers did not differ between treatments (Table 8). However, feed- ing LO resulted in higher percentage of trans $13+14$ 18:1, trans 15-18:1, and trans 16-18:1 compared with other treatments. Yield of trans $13+14$ was more than $10 \mathrm{~g} / \mathrm{d}$ higher in response to LO compared with $\mathrm{FO}$ and SO. Both FO and SO increased percentage and yield of trans 10-18:1 compared with LO. Feeding SO also resulted in higher percentage of trans $6+7+8-18: 1$ and trans9-18:1. Contrary to total trans-18:1, percentage 
Table 8. Percentage of all 18:1 isomers and yield of selected isomers in milk fat from Holstein cows fed a high-concentrate diet supplemented with fish oil (FO, 2.5\% of DM), linseed oil (LO, 5\%), or sunflower oil $(\mathrm{SO}, 5 \%)$.

\begin{tabular}{|c|c|c|c|c|c|}
\hline \multirow[b]{2}{*}{ Isomer } & \multicolumn{3}{|c|}{ Supplement } & \multirow[b]{2}{*}{ SEM } & \multirow[b]{2}{*}{$P=$} \\
\hline & $\mathrm{FO}$ & LO & $\mathrm{SO}$ & & \\
\hline & & of total $\mathrm{f}$ & & & \\
\hline \multicolumn{6}{|l|}{ 18:1 trans } \\
\hline 4 & 0.05 & 0.06 & 0.10 & 0.02 & 0.53 \\
\hline 5 & 0.05 & 0.07 & 0.11 & 0.03 & 0.25 \\
\hline $6+7+8$ & $0.51^{\mathrm{b}}$ & $0.73^{\mathrm{ab}}$ & $0.96^{\mathrm{a}}$ & 0.15 & 0.05 \\
\hline 9 & $0.49^{\mathrm{b}}$ & $0.56^{\mathrm{ab}}$ & $0.69^{\mathrm{a}}$ & 0.06 & 0.05 \\
\hline 10 & $6.16^{\mathrm{a}}$ & $1.65^{\mathrm{b}}$ & $6.47^{\mathrm{a}}$ & 1.35 & 0.05 \\
\hline 11 & 6.58 & 5.43 & 4.22 & 0.80 & 0.20 \\
\hline 12 & 0.77 & 0.96 & 0.98 & 0.13 & 0.50 \\
\hline $13+14$ & $0.88^{\mathrm{b}}$ & $3.00^{\mathrm{a}}$ & $1.27^{\mathrm{b}}$ & 0.41 & 0.05 \\
\hline 15 & $0.33^{\mathrm{b}}$ & $1.27^{\mathrm{a}}$ & $0.46^{\mathrm{b}}$ & 0.35 & 0.05 \\
\hline 16 & $0.16^{\mathrm{c}}$ & $0.97^{\mathrm{a}}$ & $0.43^{\mathrm{b}}$ & 0.12 & 0.03 \\
\hline Total & 15.7 & 13.4 & 15.2 & 2.6 & 0.77 \\
\hline \multicolumn{6}{|l|}{$18: 1 \mathrm{cis}$} \\
\hline 9 & $7.03^{\mathrm{b}}$ & $17.7^{\mathrm{a}}$ & $17.9^{\mathrm{a}}$ & 1.29 & 0.01 \\
\hline 11 & $1.64^{\mathrm{a}}$ & $0.75^{\mathrm{b}}$ & $0.82^{\mathrm{b}}$ & 0.20 & 0.01 \\
\hline 12 & $0.26^{\mathrm{b}}$ & $0.74^{\mathrm{a}}$ & $0.87^{\mathrm{a}}$ & 0.15 & 0.05 \\
\hline 13 & $0.12^{\mathrm{b}}$ & $0.23^{\mathrm{a}}$ & $0.14^{\mathrm{b}}$ & 0.02 & 0.05 \\
\hline 15 & $0.29^{\mathrm{b}}$ & $1.43^{\mathrm{a}}$ & $0.17^{\mathrm{b}}$ & 0.20 & 0.04 \\
\hline Total & $9.68^{b}$ & $22.1^{\mathrm{a}}$ & $20.4^{\mathrm{a}}$ & 1.96 & 0.01 \\
\hline \multicolumn{6}{|l|}{ Yield, g/d } \\
\hline Total trans-18:1 & 89.9 & 77.9 & 80.6 & 21.1 & 0.85 \\
\hline trans $10-18: 1$ & $31.9^{\mathrm{a}}$ & $6.96^{\mathrm{b}}$ & $26.4^{\mathrm{a}}$ & 7.1 & 0.05 \\
\hline trans $11-18: 1$ & 40.3 & 39.4 & 31.4 & 14.4 & 0.54 \\
\hline trans $13+14-18: 1$ & $5.78^{\mathrm{b}}$ & $17.1^{\mathrm{a}}$ & $7.41^{\mathrm{b}}$ & 2.9 & 0.05 \\
\hline Total cis-18:1 & $59.0^{\mathrm{b}}$ & $132.2^{\mathrm{a}}$ & $130.2^{\mathrm{a}}$ & 31.5 & 0.05 \\
\hline cis $9-18: 1$ & $42.6^{\mathrm{b}}$ & $108.7^{\mathrm{a}}$ & $116.6^{\mathrm{a}}$ & 28.1 & 0.05 \\
\hline cis $11-18: 1$ & $9.67^{\mathrm{a}}$ & $4.09^{\mathrm{b}}$ & $4.32^{\mathrm{b}}$ & 0.95 & 0.01 \\
\hline cis $15-18: 1$ & $1.96^{\mathrm{b}}$ & $7.68^{\mathrm{a}}$ & $0.94^{\mathrm{b}}$ & 1.35 & 0.03 \\
\hline
\end{tabular}

${ }^{\text {a,b,c }}$ Means with unlike superscripts differ significantly $(P<0.05)$.

and yield of total cis-18:1 isomers was greater when $\mathrm{LO}$ and SO were fed compared with FO. This response was due primarily to a 2.5 -fold decrease in the percentage and yield of cis9-18:1. In contrast, FO more than doubled the percentage and yield of cis11-18:1 and decreased cis12-18:1 compared with other treatments. Feeding LO resulted in a substantial increase in percentage and yield of cis15-18:1 compared with FO and SO.

\section{Percentage and Yield of 18:2 and 18:3 Isomers}

Similar to rumen fluid and plasma, dietary LO increased percentage and yield of total 18:3 isomers to the largest extent, primarily due to greater percentage and yield of cis9,cis12,cis15-18:3 (Table 9). Percentage and yield of total conjugated 18:2 isomers did not differ among treatments. Among conjugated 18:2 isomers, however, percentage of trans 11 ,cis $13+$ cis 9 ,cis $11-18: 2$ and trans11,trans 13-18:2 were greater in response to LO compared with other treatments. Dietary SO increased percentage and yield of trans10,cis12-18:2. Percentage and yield of cis9,trans11-18:2 did not differ due to treatment. Percentage and yield of nonconjugated 18:2 isomers increased when cows were fed LO compared with FO and SO. This response was primarily associated with greater percentage and yield of trans11,cis15-18:2, but also greater percentage of cis 9 ,trans12-18:2 and cis9, trans13-18:2. Feeding FO also resulted in greater percentage and yield of trans 11 ,cis 15 18:2 compared with SO.

\section{DISCUSSION}

\section{Milk Fat Content, Saturated Fatty Acids, and Oleic Acid}

High dietary concentrate:forage ratio and unsaturated vegetable oils (e.g., corn, soybean, linseed) reduce milk fat content and yield substantially compared with typical diets (Griinari et al., 1998; Piperova et al., 2000; Peterson et al., 2003; Loor et al., 2005a). In the present study, we did not observe a significant oil effect on milk fat content or yield despite differences, according to oil used, in percentages and yields of fatty acids synthesized de novo: (8:0-14:0), 16:0 (increased with FO), or 
Table 9. Percentage of all 18:2 and 18:3 isomers and yield of selected isomers in milk fat from Holstein cows fed a high-concentrate diet supplemented with fish oil (FO, 2.5\% of DM), linseed oil (LO, 5\%), or sunflower oil (SO, 5\%).

\begin{tabular}{|c|c|c|c|c|c|}
\hline \multirow[b]{2}{*}{ Isomer } & \multicolumn{3}{|c|}{ Supplement } & \multirow[b]{2}{*}{ SEM } & \multirow[b]{2}{*}{$P=$} \\
\hline & FO & LO & SO & & \\
\hline & $\longrightarrow$ & $f$ total $\mathrm{f}$ & 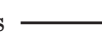 & & \\
\hline \multicolumn{6}{|l|}{$18: 3$} \\
\hline cis 9, cis 12, cis 15 & $0.60^{\mathrm{b}}$ & $1.18^{\mathrm{a}}$ & $0.66^{\mathrm{b}}$ & 0.08 & 0.01 \\
\hline $\operatorname{trans} 9, \operatorname{trans} 12, \operatorname{trans} 15$ & $0.27^{\mathrm{a}}$ & $0.27^{\mathrm{a}}$ & $0.17^{\mathrm{b}}$ & 0.02 & 0.05 \\
\hline Total & $0.94^{\mathrm{b}}$ & $1.50^{\mathrm{a}}$ & $0.89^{\mathrm{b}}$ & 0.13 & 0.05 \\
\hline \multicolumn{6}{|l|}{ 18:2 conjugated } \\
\hline cis 9, trans 11 & 2.29 & 2.36 & 1.83 & 0.25 & 0.26 \\
\hline $\operatorname{trans} 9$, cis 11 & 0.09 & 0.06 & 0.12 & 0.03 & 0.40 \\
\hline $\operatorname{trans} 10$, cis 12 & $0.03^{\mathrm{b}}$ & $0.04^{\mathrm{b}}$ & $0.14^{\mathrm{a}}$ & 0.03 & 0.06 \\
\hline cis 10, trans 12 & 0.05 & 0.02 & 0.03 & 0.01 & 0.49 \\
\hline cis 11, trans 13 & 0.04 & 0.02 & 0.03 & 0.02 & 0.91 \\
\hline trans $11, \operatorname{cis} 13+\operatorname{cis} 9, \operatorname{cis} 11$ & $0.05^{\mathrm{b}}$ & $0.10^{\mathrm{a}}$ & $0.06^{\mathrm{b}}$ & 0.02 & 0.05 \\
\hline trans 11, trans 13 & $0.08^{\mathrm{b}}$ & $0.15^{\mathrm{a}}$ & $0.08^{\mathrm{b}}$ & 0.03 & 0.05 \\
\hline trans,trans & 0.11 & 0.09 & 0.13 & 0.04 & 0.90 \\
\hline Total & 2.73 & 2.84 & 2.40 & 0.21 & 0.40 \\
\hline \multicolumn{6}{|l|}{ 18:2 nonconjugated } \\
\hline cis $9, \operatorname{cis} 12$ & $1.64^{\mathrm{b}}$ & $2.10^{\mathrm{b}}$ & $3.57^{\mathrm{a}}$ & 0.24 & 0.01 \\
\hline cis 9, trans 12 & $0.07^{\mathrm{c}}$ & $0.24^{\mathrm{a}}$ & $0.16^{\mathrm{b}}$ & 0.03 & 0.05 \\
\hline $\operatorname{trans} 8, \operatorname{cis} 13$ & 0.15 & 0.16 & 0.16 & 0.03 & 0.99 \\
\hline $\operatorname{trans} 9, \operatorname{cis} 12$ & 0.16 & 0.22 & 0.10 & 0.04 & 0.19 \\
\hline cis 9, trans 13 & $0.32^{\mathrm{b}}$ & $0.99^{\mathrm{a}}$ & $0.41^{\mathrm{b}}$ & 0.13 & 0.05 \\
\hline $\operatorname{trans} 9, \operatorname{trans} 12$ & 0.15 & 0.16 & 0.07 & 0.05 & 0.21 \\
\hline trans 11, cis 15 & $1.75^{\mathrm{b}}$ & $2.87^{\mathrm{a}}$ & $0.41^{\mathrm{c}}$ & 0.17 & 0.01 \\
\hline Total & $4.33^{\mathrm{b}}$ & $6.82^{\mathrm{a}}$ & $4.98^{\mathrm{b}}$ & 0.60 & 0.05 \\
\hline \multicolumn{6}{|l|}{ Yield, g/d } \\
\hline Total nonconjugated 18:2 & $26.0^{\mathrm{b}}$ & $40.0^{\mathrm{a}}$ & $28.3^{\mathrm{b}}$ & 6.6 & 0.05 \\
\hline trans $11, \operatorname{cis} 15-18: 2$ & $10.2^{\mathrm{b}}$ & $17.6^{\mathrm{a}}$ & $1.82^{\mathrm{c}}$ & 2.77 & 0.05 \\
\hline Total conjugated $18: 2$ & 17.0 & 17.9 & 15.6 & 5.8 & 0.74 \\
\hline cis 9, trans11-18:2 & 14.2 & 15.6 & 13.0 & 5.7 & 0.83 \\
\hline trans 10, cis $12-18: 2$ & $0.15^{\mathrm{b}}$ & $0.12^{\mathrm{b}}$ & $0.57^{\mathrm{a}}$ & 0.10 & 0.05 \\
\hline Total 18:3 & $6.06^{\mathrm{ab}}$ & $8.34^{\mathrm{a}}$ & $5.03^{\mathrm{b}}$ & 2.05 & 0.05 \\
\hline $18: 3 n-3$ & $3.74^{\mathrm{b}}$ & $7.04^{\mathrm{a}}$ & $3.77^{\mathrm{b}}$ & 1.29 & 0.05 \\
\hline
\end{tabular}

a,b,c Means with unlike superscripts differ significantly $(P<0.05)$.

taken up from the blood: 18:0, and cis9-18:1 (decreased with FO). However, the low level of milk fat content observed with LO in this study is in agreement with previous results (Loor et al., 2005a).

The marked reduction in milk stearic acid yield with fish oil was associated with an apparent reduction in biohydrogenation of trans-18:1 to $18: 0$ as shown by lower ruminal 18:0 percentage (Table 3) and duodenal flow (Loor et al., 2005c). Indeed, ruminal concentrations of trans-18:1 (Table 3), although numerically lower, did not differ significantly between FO (22.6\%), LO (24.7\%), or SO (25.6\%), despite more than 2-fold lower intake of 18:2n-6 and 18:3n-3 in cows fed FO (Loor et al., 2005b). Intake of 18:0 across diets was low, and with FO was $\sim 18 \mathrm{~g} / \mathrm{d}$ lower (19 vs. $37 \mathrm{~g} / \mathrm{d}$; Loor et al., 2005b). Thus, it is likely that dietary 18:0 intakes had a modest contribution to its ruminal concentration. Reduced ruminal outflow of 18:0 derived from biohydrogenation of unsaturated fatty acids likely reduced availability of 18:0 (low plasma concentration; Table 4) for desaturation, resulting in lower milk yield of cis9-18:1. Based on ex- tensive literature and current results, availability of 18:0 for endogenous cis9-18:1 production seems to be crucial for milk fat synthesis (see also Donovan et al., 2000; Chilliard et al., 2003; Loor et al., 2005b). Furthermore, the reduction in 18:0 availability seems to be the strongest link among nearly all previous studies dealing with milk fat depression induced by fish oil supplementation (see review by Chilliard et al., 2001; Donovan et al., 2000; Shingfield et al., 2003; Loor et al., 2005b).

Interestingly, we observed a significant negative correlation ( $\mathrm{r}=-0.73, P=0.02$ ) between ruminal fluid cis918:1 and milk fat content (Figure 1C). This negative relationship could be due in part to the fact that oleic acid in the rumen can be isomerized to trans10-18:1 (Mosley et al., 2002) [which tended $(P=0.12)$ to be negatively correlated $(\mathrm{r}=-0.55)$ with milk fat content], and is supported by a positive correlation $(\mathrm{r}=0.60, P=$ 0.08 ) between both fatty acids in the rumen (Figure $1 \mathrm{~F})$. Ruminal oleic acid also was positively correlated $(\mathrm{r}=0.63, P=0.07)$ with ruminal trans9, trans 12-18:2, 

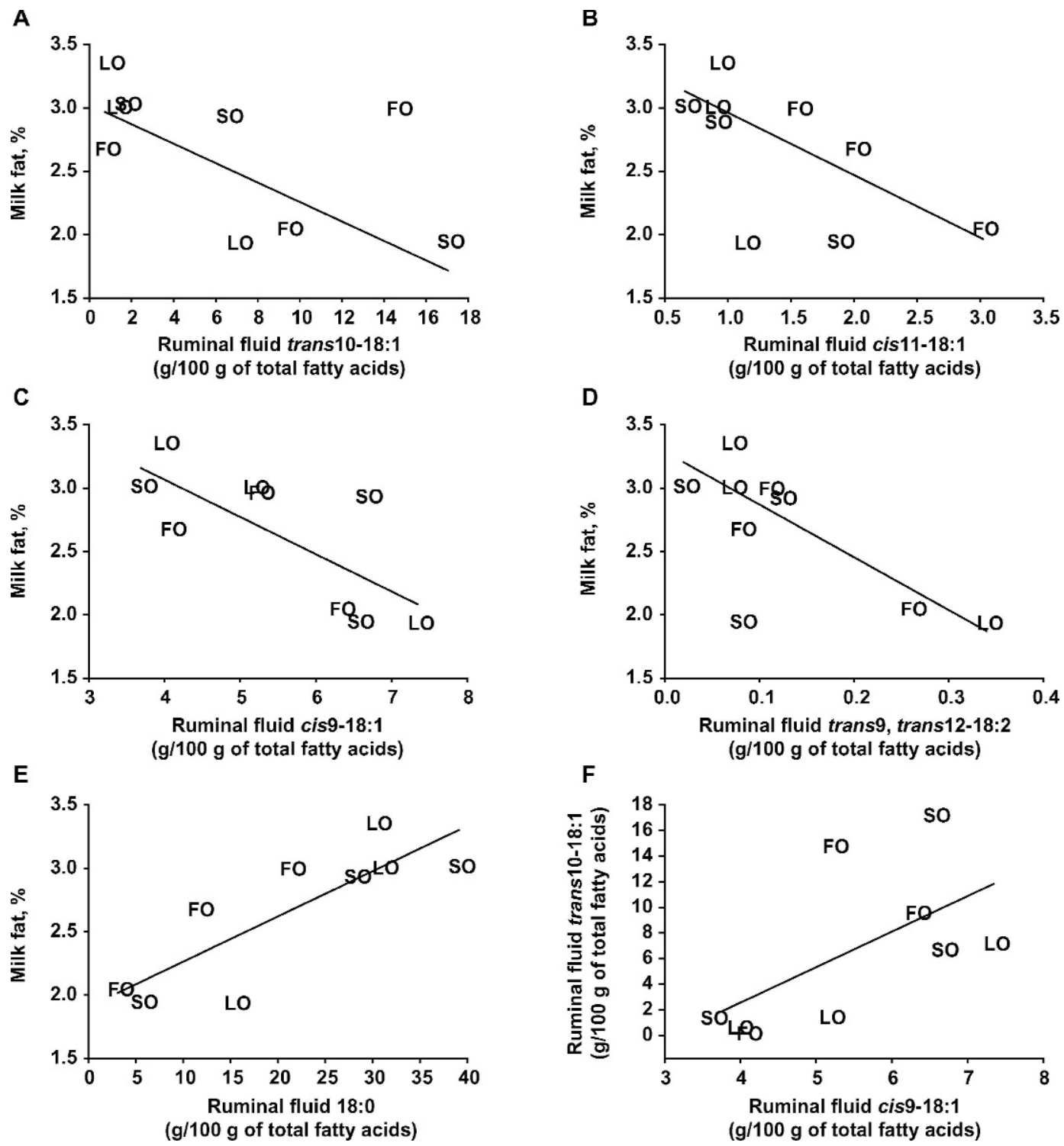

Figure 1. Correlation analysis among ruminal fluid concentration of selected fatty acids and milk fat content or among ruminal concentration of cis9-18:1 and trans10-18:1. Correlation values were $-0.55(P=0.12),-0.61(P<0.05),-0.73(P<0.01),-0.70(P<0.01),+0.82(P<$ $0.01)$, and $+0.60(P<0.05)$ for panels A through $\mathrm{F}$.

which, based on our previous analysis (Loor et al., 2005a) and current data (Figure 1D), is negatively associated with milk fat content. Intake of oleic acid in this study was $124 \mathrm{~g} / \mathrm{d}$ lower in cows fed FO compared with SO and LO (Loor et al., 2005c), but there were no differences in ruminal fluid cis9-18:1 percentage across diets (Table 2), which raises the possibility that, in cows fed FO, other 18:1 and(or) 18:2 intermediates gave rise to oleic acid. In this context, the negative correlation $(\mathrm{r}=$ $(0.71, \mathrm{P}<0.05)$ between ruminal oleic acid and trans 11 18:1 suggests the latter could be isomerized to cis9-18:1 (Mosley et al., 2002). Despite the negative correlation between ruminal oleic acid and milk fat content, data do not lend support to this fatty acid as being involved in the low-fat milk syndrome because 1) there was no correlation $(\mathrm{r}=-0.08)$ between milk fat cis $9-18: 1$ and milk fat content and 2) ruminal cis9-18:1 was not significantly correlated with its percentage in milk fat $(\mathrm{r}=0.20)$.

Data from this study suggest that feeding high-concentrate diets with linseed oil and sunflower oil may result in a more pronounced decrease in de novo fatty acid synthesis than fish oil, which would partly account for the observation that the 3 oils resulted in milk with low fat content. Thus, a decrease in synthesis of 8:016:0 (resulting in their lower percentage in milk fat 
with both LO and SO, Table 7) could be a more important cause of milk fat depression with either 18:3(Loor et al., 2005a) or 18:2-rich oil supplementation, than with FO supplementation.

\section{Trans10-18:1, Cis11-18:1, Nonconjugated Isomers, and Milk Fat Depression}

We previously compiled literature data confirming (Griinari et al., 1998; Bauman and Griinari, 2003) that increased percentage of trans 10-18:1 in milk fat is positively correlated with milk fat depression in cows fed high-concentrate diets with or without unsaturated oils, or mixed diets with various levels of fish oil (Loor et al., 2005a). In the present study, we found that FO and SO compared with LO, resulted in 2.7-fold increase in trans10-18:1 in rumen, a 3-fold increase in plasma, and a 4-fold increase in milk. Despite the similar low level of milk fat content and yield among the 3 diets, ruminal trans $10-18: 1$ tended $(P=0.12)$ to be negatively correlated with milk fat content. Our data indicate that low milk fat content when feeding FO is associated with appreciable increases in cis 11-18:1 in ruminal fluid (2.1fold), plasma (2.1-fold), and milk fat (2- to 3-fold). We had previously observed a 2-fold increase in cis11-18:1 percentage in milk fat along with a $28 \%$ reduction in milk fat yield in cows receiving a $300-\mathrm{mL}$ infusion of fish oil into the rumen compared with controls (Loor et al., 2005b). Data from Offer et al. (1999), Donovan et al. (2000), Whitlock et al. (2002), and Shingfield et al. (2003) provide other evidence for a link between cis1118:1 and FO-induced milk fat depression. Dietary FO resulted in a 7-fold increase in percentage of trans9,cis11-18:2 in rumen (Table 3), suggesting this fatty acid may be a ruminal precursor of cis11-18:1. Interestingly, concentration of trans 9, cis 11-18:2 in plasma was 12 -fold greater with FO (Table 5) compared with $\mathrm{LO}$ or SO, but its concentration in milk fat did not differ (Table 8). The relatively high concentrations of trans11,cis15-18:2 in rumen, plasma, and milk with FO could also contribute to low milk fat yield and content (see Loor et al., 2005a).

Ruminal percentage of trans9,trans 12-18:2 and trans 9 ,cis $12-18: 2$ were negatively correlated with milk fat content, which agrees with our previous study with LO supplementation (Loor et al., 2005a). Although their concentrations in plasma and percentages in milk fat did not differ due to oils in the present study, both isomers increased in ruminal fluid with LO (Table 2). The negative relationship between rumen percentage and milk fat content (Figure 1D) for trans9,trans 12 18:2 ( $\mathrm{r}=-0.69 ; P=0.05)$ suggests that this isomer might be a component of the mechanisms whereby 18:3rich oils cause milk fat depression. Responses to feeding
LO were marked also for percentages of several other 18:3n-3 biohydrogenation intermediates: trans $13+14$ 18:1, trans $15-18: 1$, trans $16-18: 1$, cis $15-18: 1$, cis9,trans 12-18:2, and trans 11,cis15-18:2 in rumen, plasma, and milk fat, and cis9,trans13-18:2 in plasma and milk (likely arising from desaturation of trans 13-18:1). In a previous study, milk fat cis 15-18:1, cis9,trans12-18:2, cis9,trans13-18:2, and trans11,cis15-18:2 were negatively correlated with milk fat 4:0-16:0 percentage (Loor et al., 2005a), and are other candidates to explain the decrease in de novo lipogenesis with LO.

Although abomasal infusions of trans 10,cis 12-18:2 or dietary rumen-inert mixtures of CLA have caused milk fat depression (Baumgard et al., 2000; Loor and Herbein, 2003; Perfield et al., 2004), feeding studies are inconclusive on the putative role of nondietary, endogenously synthesized trans10,cis 12-18:2. Increases in milk fat trans10,cis12-18:2 in cows with diet-induced milk fat depression were very modest (less than 0.10 to $0.15 \%$ of total milk fatty acids; Piperova et al., 2000; Whitlock et al., 2002; Peterson et al., 2003; present study). Even with polyunsaturated fatty acid supplementation, results show discrepancies between trans10,cis 12-18:2 and milk fat yield (AbuGhazaleh et al., 2003; Shingfield et al., 2003; Loor et al., 2005a,b) or even no correlation between milk trans10,cis12-18:2 and milk fat content (Precht et al., 2002; Bradford and Allen, 2004; Loor et al., 2005a; present study). It appears likely that other biohydrogenation intermediates in addition to trans10,cis12-18:2 are involved in dietinduced milk fat depression (Bauman and Griinari, 2003; Peterson et al., 2003; Loor et al., 2005a,b).

\section{Biohydrogenation Intermediates with Practical Applications}

Responses in the percentage and yield of certain fatty acids with potential applications in human health and energy sparing in the cow (Bauman and Griinari, 2003) differed according to polyunsaturated fatty acid supply in the diet. Although we did not include a control diet for comparison, all oils resulted in high trans11-18:1 and cis9,trans11-18:2 in milk fat, which are far above typical values in milk from cows receiving nonlipidsupplemented diets. Comparison of our measurements of duodenal flow of trans 11-18:1 and cis9,trans 11-18:2 (Loor et al., 2004b; 2005c) with their yield in milk fat across diets confirmed (Piperova et al., 2002; Shingfield et al., 2003) that endogenous synthesis is the primary source of cis9,trans11-18:2. Even if all of the duodenal cis9,trans11-18:2 was secreted in milk fat, we observed that when feeding FO, LO, or SO, at least 97,90 , or $82 \%$, respectively, of milk cis9,trans11-18:2 was synthesized via trans11-18:1 desaturation. These estimates are 
lower than previous ones (Piperova et al., 2002) and suggest for the first time that endogenous synthesis of cis9,trans 11-18:2 may decrease as duodenal flow of this isomer increases. More importantly, we present evidence that supplementation of a high-concentrate diet with fish oil providing $<25 \% \mathrm{EPA}$ and DHA relative to linoleic acid from sunflower oil was effective in enhancing flow of trans11-18:1 to maintain synthesis of cis9,trans11-18:1 in the mammary gland. This result confirms in vitro data showing similar accumulation of trans-18:1 due to DHA compared with soybean oil at 6 times the level of DHA supplementation (AbuGhazaleh and Jenkins, 2004).

Previous studies have observed increases in milk trans10-18:1 with fish oil supplementation to TMR (Donovan et al., 2000; Whitlock et al., 2002; Shingfield et al., 2003) or fish oil infusion into the rumen in cows fed corn silage diets (Loor et al., 2005b). Our data suggest that it is feasible to enhance ruminal outflow of trans11-18:1 and simultaneously trans10-18:1, cis1118:1, or trans 11 ,cis $15-18: 2$ by supplementing fish oil at a fraction of linoleic acid-rich oils when fed in combination with high-concentrate diets. The net result is greater trans11-18:1 and cis9,trans 11-18:2 in milk and potentially more trans $10-18: 1$, cis $11-18: 1$, or trans11,cis15-18:2 to reduce milk fat synthesis. This could be an effective strategy to reduce milk fat output and spare energy, which may be useful in certain situations in high-yielding dairy cows (Bauman and Griinari, 2003), while enhancing concentration of the anticarcinogenic cis9,trans11-18:2 (Corl et al., 2003). However, there is a need to evaluate how the increase in several other trans isomers of 18:1, 18:2, or 18:3 (Tables 8 and 9 ) could positively or negatively affect the nutritional value of milk fat.

In summary, milk fat content did not differ among oil treatments but was clearly lower when compared with feeding high- or low-concentrate diets alone (Loor et al., 2005a). Trans10,cis12-18:2 increased in rumen or milk fat with sunflower oil, but percentage and yield of trans 10-18:1 was similar among fish oil and sunflower oil. Only feeding SO led to changes typical for classical diet-induced milk fat depression, i.e., increased trans10-18:1 and trans10,cis12-18:2 and decreased de novo fatty acid synthesis. Fish oil resulted in a marked increase in percentage of cis11-18:1 in rumen, plasma, and milk fat. The effects of FO, compared with LO and SO, on milk fat were not mediated via inhibition of de novo fatty acid synthesis. Results with LO confirmed previous data (Loor et al., 2005a) for higher trans $13+14-18: 1$, trans $15-18: 1$, trans 16-18:1, cis 15-18:1, cis9,trans $12-18: 2$, cis 9 ,trans $13-18: 2$ and trans11,cis15-18:2. Although persistent, increases in cis11-18:1, trans 11,cis 15-18:2, and trans9,trans 12-18:2 with both $\mathrm{LO}$ and $\mathrm{FO}$ occurred along with the increase in other isomers in ruminal fluid and milk fat, making it difficult to differentiate their respective roles in dietinduced milk fat depression. Taken together, our data indicates that milk fat depression typically observed with fish oil supplementation could be linked to reduced availability of stearic acid and a potential antilipogenic effect of trans10-18:1, cis11-18:1, or trans11,cis15-18:2. Simultaneous increases in ruminal trans11-18:1, with fish oil at a fraction of sunflower oil supplementation, may represent an effective strategy to maintain cis9,trans11-18:2 synthesis in mammary while reducing milk fat output and sparing energy. Feeding studies are still inconclusive on the role of ruminally-derived trans 10,cis12-18:2 and milk fat depression but highlight the possibility that other biohydrogenation intermediates may also play a role in this phenomenon.

\section{ACKNOWLEDGMENTS}

Authors acknowledge the assistance of J. P. Pezant and the team of Les Cèdres Experimental Unit for feeding, milking, blood sampling, and caring for cows, as well as M. Tourret, and C. Delavaud for help in laboratory analyses. This study was partly funded by the INRA Programme "Micronutrients in animal products, and their health value for consumers."

\section{REFERENCES}

AbuGhazaleh, A. A., and T. C. Jenkins. 2004. Short communication: Docosahexaenoic acid promotes vaccenic acid accumulation in mixed ruminal cultures incubated with linoleic acid. J. Dairy Sci. $87: 645-651$.

AbuGhazaleh, A. A., D. J. Schingoethe, A. R. Hippen, and K. F. Kalscheur. 2003. Milk conjugated linoleic acid response to fish oil supplementation of diets differing in fatty acid profiles. J. Dairy Sci. 86:944-953.

Association of Official Analytical Chemists. 1997. Official Methods of Analysis. 16th ed. AOAC Int., Gaithersburg, MD.

Bauman, D. E., and J. M. Griinari. 2003. Nutritional regulation of milk fat synthesis. Annu. Rev. Nutr. 23:203-227.

Baumgard, L. H., B. A. Corl, D. A. Dwyer, A. Sæbø, and D. E. Bauman. 2000. Identification of the conjugated linoleic acid isomer that inhibits milk fat synthesis. Am. J. Physiol. 278:R179-R184.

Bonnet, M., C. Delavaud, K. Laud, I. Gourdou, C. Leroux, J. Djiane, and Y. Chilliard. 2002. Mammary leptin synthesis, milk leptin and their putative physiological roles. Reprod. Nutr. Dev. 42:399-413.

Bradford, B. J., and M. S. Allen. 2004. Milk fat responses to a change in diet fermentability vary by production level in dairy cattle. J. Dairy Sci. 87:3800-3807.

Chilliard, Y., and A. Ferlay. 2004. Dietary lipids and forages interactions on cow and goat milk fatty acid composition and sensory properties. Reprod. Nutr. Dev. 44:467-492.

Chilliard, Y., A. Ferlay, and M. Doreau. 2001. Effect of different types of forages, animal fat or marine oils in cow's diet on milk fat secretion and composition, especially conjugated linoleic acid (CLA) and polyunsaturated fatty acids. Livest. Prod. Sci. 70:31-48.

Chilliard, Y., J. Rouel, P. Capitan, J. M. Chabosseau, K. RaynalLjutovac, and A. Ferlay. 2003. Correlations between milk fat 
content and fatty acid composition in goats receiving different combinations of forages and lipid supplements. Page 343 in Book of Abstracts, 54th Annu. Meeting EAAP, Rome, Italy. Y. van der Honing, ed. Wageningen Acad. Publ., Wageningen, The Netherlands.

Corl, B. A., D. M. Barbano, D. E. Bauman, and C. Ip. 2003. Cis-9, trans-11 CLA derived endogenously from trans-11 18:1 reduces cancer risk in rats. J. Nutr. 133:2893-2900.

Delavaud, C., A. Ferlay, Y. Faulconnier, F. Bocquier, G. Kann, and Y. Chilliard. 2002. Plasma leptin concentration in adult cattle: Effects of breed, adiposity, feeding level, and meal intake. J. Anim. Sci. 80:1317-1328.

Donovan, D. C., D. J. Schingoethe, R. J. Baer, J. Ryali, A. R. Hippen, and S. T. Franklin. 2000. Influence of dietary fish oil on conjugated linoleic acid and other fatty acids in milk fat from lactating dairy cows. J. Dairy Sci. 83:2620-2628.

Doreau, M., and Y. Chilliard. 1997. Effects of ruminal or postruminal fish oil supplementation on intake and digestion in dairy cows. Reprod. Nutr. Dev. 37:113-124.

Ferlay, A., and Y. Chilliard. 1999. Effects of the infusion of nonselective beta-, and selective beta1- or beta2-adrenergic agonists, on body fat mobilisation in underfed or overfed non-pregnant heifers. Reprod. Nutr. Dev. 39:409-421.

Griinari, J. M., D. A. Dwyer, M. A. McGuire, D. E. Bauman, D. L. Palmquist, and K. V. V. Nurmela. 1998. Trans-octadecenoic acids and milk fat depression in lactating cows. J. Dairy Sci. 81:1251-1261.

Loor, J. J., M. Doreau, J. M. Chardigny, A. Ollier, J. L. Sebedio, and Y. Chilliard. 2005b. Effects of ruminal or duodenal supply of fish oil on milk fat secretion and profiles of trans-fatty acids and conjugated linoleic acid isomers in dairy cows fed maize silage. Anim. Feed Sci. Technol. 119:227-246.

Loor, J. J., A. Ferlay, A. Ollier, M. Doreau, and Y. Chilliard. 2005a. Relationship among trans and conjugated fatty acids and bovine milk fat yield due to dietary concentrate and linseed oil. J. Dairy Sci. 88:726-740.

Loor, J. J., and J. H. Herbein. 2003. Reduced fatty acid synthesis and desaturation due to exogenous trans10,cis12-CLA in cows fed oleic or linoleic oil. J. Dairy Sci. 86:1354-1369.

Loor, J. J., K. Ueda, A. Ferlay, Y. Chilliard, and M. Doreau. 2004a. Short communication: Diurnal profiles of conjugated linoleic acids and trans fatty acids in ruminal fluid from cows fed a highconcentrate diet supplemented with fish oil, linseed oil, or sunflower oil. J. Dairy Sci. 87:2468-2471.

Loor, J. J., K. Ueda, A. Ferlay, Y. Chilliard, and M. Doreau. 2004b. Biohydrogenation, duodenal flows, and intestinal digestion of trans fatty acids and conjugated linoleic acids in response to dietary forage:concentrate ratio and linseed oil in dairy cows. J. Dairy Sci. 87:2472-2485.

Loor, J. J., K. Ueda, A. Ferlay, Y. Chilliard, and M. Doreau. 2005c. Intestinal flow and digestibility of trans fatty acids and conjugated linoleic acids (CLA) in dairy cows fed a high-concentrate diet supplemented with fish oil, linseed oil, or sunflower oil. Anim. Feed Sci. Technol. 119:203-225.
Mosley, E. E., G. Powell, M. Riley, and T. C. Jenkins. 2002. Microbial biohydrogenation of oleic acid to trans isomers in vitro. J. Lipid Res. 43:290-296.

Oba, M., and M. S. Allen. 2003. Effects of corn grain conservation method on feeding behavior and productivity of lactating dairy cows at two dietary starch concentrations. J. Dairy Sci. 86:174-183.

Offer, N. W., M. Marsden, J. Dixon, B. K. Speake, and F. E. Thacker. 1999. Effect of dietary fat supplements on levels of $n-3$ polyunsaturated fatty acids, trans acids and conjugated linoleic acid in bovine milk. Anim. Sci. 69:613-625.

Perfield, J. W., II, A. L. Lock, A. M. Pfeiffer, and D. E. Bauman. 2004. Effects of amide-protected and lipid-encapsulated conjugated linoleic acid supplements on milk fat synthesis. J. Dairy Sci. 87:3010-3016.

Peterson, D. G., E. A. Matitashvili, and D. E. Bauman. 2003. Dietinduced milk fat depression in dairy cows results in increased trans-10, cis-12 CLA in milk fat and coordinate suppression of mRNA abundance for mammary enzymes involved in milk fat synthesis. J. Nutr. 133:3098-3102.

Piperova, L. S., J. Sampugna, B. B. Teter, K. Kalscheur, M. Yurawecz, Y. Ku, K. Morehouse, and R. A. Erdman. 2002. Duodenal and milk trans octadecenoic acid and conjugated linoleic acid (CLA) isomers indicate postabsorptive synthesis is the predominant source of cis9-containing CLA in lactating dairy cows. J. Nutr. 132:1235-1241.

Piperova, L. S., B. B. Teter, I. Bruckental, J. Sampugna, S. E. Mills, M. P. Yurawecz, J. Fritsche, K. Ku, and R. A. Erdman. 2000. Mammary lipogenic enzyme activity, trans fatty acids and conjugated linoleic acids are related in lactating dairy cows fed a milk fat-depressing diet. J. Nutr. 130:2568-2574.

Precht, D., H. Hagemeister, W. Kanitz, and J. Voigt. 2002. Milk fat depression and the role of trans and CLA fatty acid isomers by feeding a high fiber diet with calcium soaps of fatty acids in early lactating dairy cows. Milchwissenschaft 57:518-522.

Roy, A., A. Ferlay, A. Ollier, and Y. Chilliard. 2005. Kinetics of trans and conjugated fatty acids (FA) concentrations in cow milk after addition of plant oils to different basal diets. Page 136 in Book of Abstracts, 56th Annu. Meeting EAAP, Uppsala, Sweden. Wageningen Acad. Publ., Wageningen, The Netherlands.

Shingfield, K., S. Ahvenjärvi, V. Toivonen, A. Ärölä, K. V. V. Nurmela, P. Huhtanen, and J. Griinari. 2003. Effect of dietary fish oil on biohydrogenation of fatty acids and milk fatty acid content in cows. Anim. Sci. 77:165-179.

Sutton, J. D., M. S. Dhanoa, S. V. Morant, J. France, D. J. Napper, and E. Schuller. 2003. Rates of production of acetate, propionate, and butyrate in the rumen of lactating dairy cows given normal and low-roughage diets. J. Dairy Sci. 86:3620-3633.

Whitlock, L. A., D. J. Schingoethe, A. R. Hippen, K. F. Kalscheur, R. J. Baer, N. Ramaswamy, and K. M. Kasperson. 2002. Fish oil and extruded soybeans fed in combination increase conjugated linoleic acids in milk of dairy cows more than when fed separately. J. Dairy Sci. 85:234-243. 